\title{
ARTICLE Short ragweed pollen promotes M2 macrophage polarization via TSLP/TSLPR/OX40L signaling in allergic inflammation
}

\author{
Ruzhi Deng ${ }^{1,2}$, Xin Chen ${ }^{1,2}$, Yun Zhang ${ }^{1,2}$, Fang Bian ${ }^{1}$, Ning Gao ${ }^{1}$, Jiaoyue Hu ${ }^{1}$, Changjun Wang ${ }^{1}$, Rodrigo G. de Souza ${ }^{1}$, Fan Lu $^{2}$, \\ Stephen C. Pflugfelder ${ }^{1}$ and De-Quan $\mathrm{Li}^{1}$
}

This study was to explore the role and mechanism of macrophages in pollen-triggered allergic inflammation. A murine model of short ragweed (SRW) pollen-induced experimental allergic conjunctivitis (EAC), and bone marrow (BM)-macrophages cultures were used. Typical allergic manifestations and TSLP-stimulated Th2 hyperresponse were observed in ocular surface of EAC model in wild-type (WT) mice induced by SRW. The M2 phenotype markers, Arg1, Ym1 and FIZZ1, were highly expressed by conjunctiva and draining cervical lymph nodes (CLNs) of WT-EAC mice when compared with controls, as evaluated by RT-qPCR and Immunofluorescent double staining with macrophage marker F4/80. The stimulated expression of TSLPR and OX40L by macrophage was detected in conjunctiva and CLNs by RT-qPCR, double staining, and flow cytometry. M2 macrophages were found to produce TARC and MDC. In contrast, EAC model with TSLPR ${ }^{-1-}$ mice did not show allergic signs and any increase of Th2 cytokines (IL-4, IL-5 and IL-13) and M2 markers. In vitro cultures confirmed that SRW extract stimulates expression of TSLPR, OX40L, TARC, MDC, and three M2 markers by BM-macrophages from WT mice, but not from TSLPR ${ }^{-1-}$ mice. These findings demonstrate that SRW pollen primes macrophage polarization toward to M2 phenotype via TSLP/TSLPR/OX40L signaling to amplify allergic inflammation.

Mucosal Immunology (2019) 12:1141-1149; https://doi.org/10.1038/s41385-019-0187-8

\section{INTRODUCTION}

Allergic diseases affect large populations worldwide with increased incidence over the past decades. As a ubiquitous allergen, pollen has been known to be an important trigger and exacerbating factor of allergic informatory diseases, including asthma, rhinitis, atopic dermatitis, and seasonal conjunctivitis. Recent investigations have made groundbreaking discoveries in mucosal immunity and the cellular and molecular mechanism by which allergic inflammation is initiated and developed through the interaction between innate immunity and adaptive responses. $^{1,2}$

The one of major advances in mucosal immunology is that epithelium-derived pro-allergic cytokines have been recognized as the master initiators in allergic inflammatory diseases, and thus allergic diseases have being proposed as epithelial disorders both structurally and functionally. Thymic stromal lymphopoietin (TSLP) and interleukin (IL)-33 are well defined epithelial pro-allergic cytokines, which initiate the T helper type 2 (Th2)-dominant allergic inflammatory disease including atopic dermatitis, asthma and allergic conjunctivitis. TSLP has been known to activate dendritic cells to produce OX40 ligand (OX40L) that primes naive $\mathrm{CD} 4+\mathrm{T}$ cells to differentiate into Th2 cells, which produce Th2 cytokines, IL-4, IL-5, and IL-13. 3,4 IL-33, identified as a functional ligand to ST2, is able to directly activate ST2 receptor on Th2 cells that mediate allergic inflammation., ${ }^{5,6}$ Our group has also revealed the TSLP/OX40L/OX40 and IL-33/ST2 allergic pathways in the murine model of experimental allergic conjunctivitis (EAC) induced by short ragweed (SRW) pollen. ${ }^{7-9}$

Besides the epithelial and dendritic cells, macrophages are essential components of innate immunity system and play a critical role in primary responses to pathogens, inflammation, and tissue repair. Intensive investigations have identified that macrophages exhibit unique activation patterns upon exposure to prototypical cytokines or TLR agonists. At least two functionally distinct subsets of macrophages have been recognized: the classical activated (M1) and alternatively activated (M2) macrophages. M1 macrophages are differentiated by Th1 cytokines such as IFN- $\gamma$, proinflammatory cytokines such as TNF- $a$, and microbial products such as lipopolysaccharide (LPS). M1 macrophages play important roles in the exacerbation of the inflammation caused by proinflammatory cytokines, and produce toxic agents that eradicate bacterial, fungal, and viral infections. As the opposite end of the macrophage spectrum, M2 macrophages decrease the inflammatory responses by suppressing Th1 cell responses. The differentiation of M2 macrophages is promoted by Th2 cytokines such as IL-4 and IL-13.10,11

M2 macrophages have been recently reported to contribute to allergic inflammation in the asthma, dermatitis, and other allergies. ${ }^{10-14}$ However, the role and underlying mechanism of macrophage in allergic inflammation by pollen has not been well elucidated. It has not been well documented whether macrophages express TSLP receptor (TSLPR) and produce OX40L in response to

\footnotetext{
${ }^{1}$ Ocular Surface Center, Cullen Eye Institute, Department of Ophthalmology, Baylor College of Medicine, Houston, TX, USA and ${ }^{2}$ School of Optometry and Ophthalmology, Wenzhou Medical University, Wenzhou, China

Correspondence: De-Quan Li (dequanl@bcm.tmc.edu)

These authors contributed equally: Ruzhi Deng, Xin Chen
}

Received: 25 January 2019 Revised: 11 June 2019 Accepted: 16 June 2019

Published online: 26 July 2019 

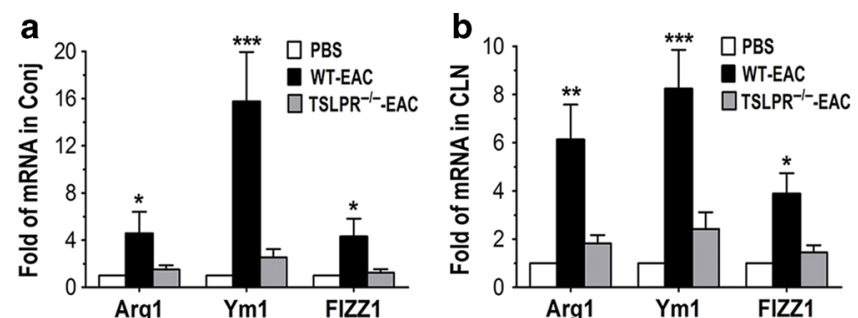

d

C PBS WT-EAC
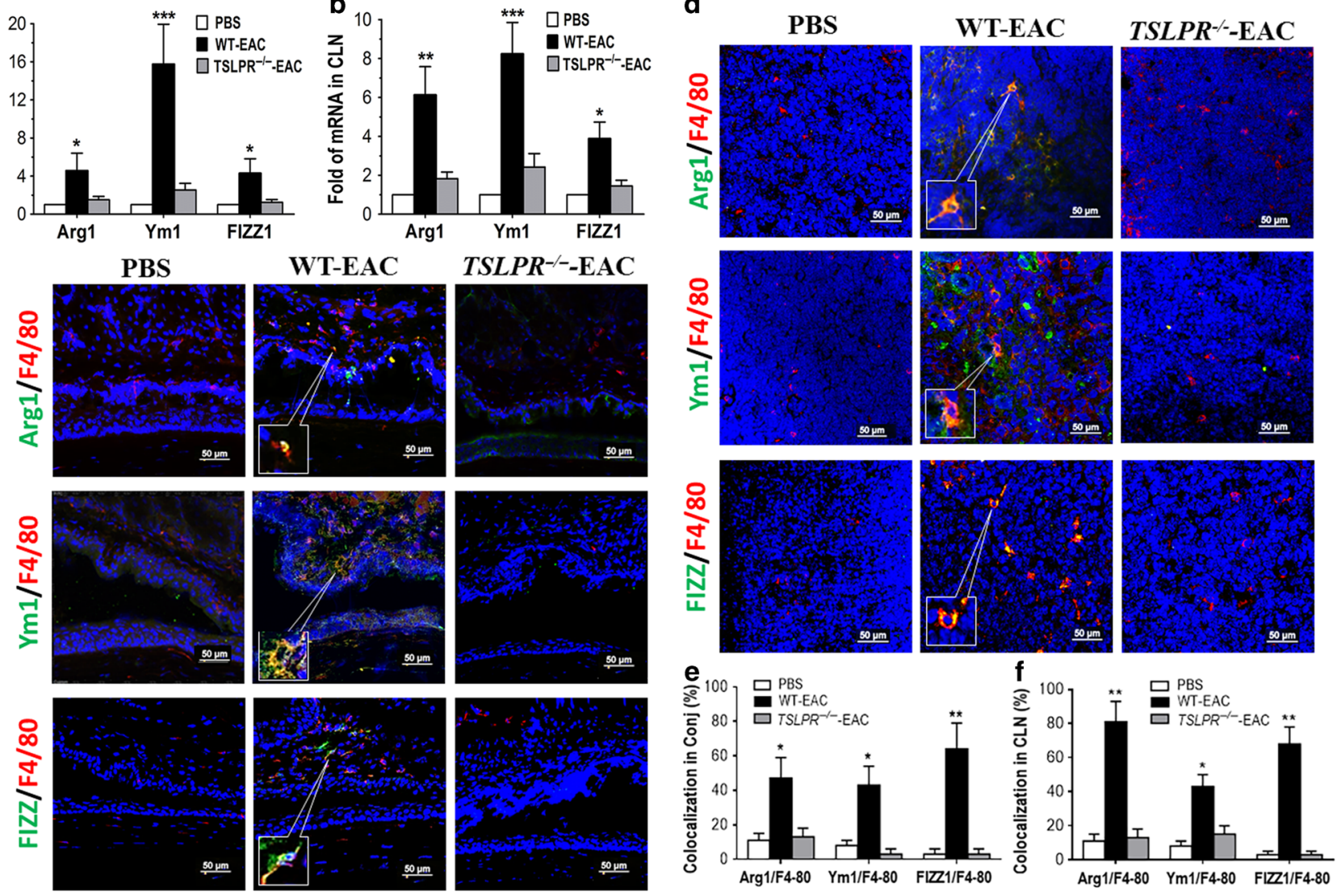

Fig. 1 M2 macrophages were identified by three markers Arg1, Ym1 and FIZZ1 in the ocular surface and draining CLNs of a murine EAC model induced by SRW pollen. $\mathbf{a}$, $\mathbf{b}$ the mRNA expression of Arg1, Ym1, and FIZZ1 in conjunctiva (Conj, a) and CLNs (b) of PBS control, WT-EAC and TSLPR ${ }^{-1-}$-EAC mice. C, d Representative images of immunofluorescent double staining of Arg1, Ym1 or FIZZ1, together with F4/80 antibody at ocular surface (c), and in CLNs (d) of PBS, WT-EAC, and TSLPR ${ }^{-1-}$-EAC mice. e, $\mathbf{f}$ The measurement of colocalization of F4/80 with Arg1, Ym1, and FIZZ1 by double staining in ocular surface (e) and CLNs (f) of PBS, WT-EAC and TSLPR ${ }^{-1-}$-EAC mice. Results shown are the Mean \pm SD. ${ }^{*} P<0.05,{ }^{* *} P<0.01,{ }^{* * *} P<0.001 ; n=5$, compared with controls

epithelial pro-allergic cytokine TSLP, similar to dendritic cells; and whether macrophages are polarized to $\mathrm{M} 2$ phenotype primed by the pollen to amplify allergic inflammation. The present study was aimed to explore the underlying mechanism by which pollen promotes M2 macrophage polarization to amplify allergic inflammation via TSLP/TSLPR/OX40L signaling in a murine model of SRW pollen-induced EAC, as well as an in vitro culture model of murine bone marrow (BM)-derived macrophages.

\section{RESULTS}

Macrophages were polarized to alternatively activated phenotype in the ocular surface and draining CLNs of a murine EAC model induced by SRW pollen

Compared with untreated and PBS treated mice as controls, the repeated topical challenges with SRW pollen allergen in SRWsensitized WT-BALB/c mice generated typical clinical manifestations similar to human allergic conjunctivitis, consistent with previous reports. ${ }^{7,8,15}$ Interestingly, we observed a significant upregulation of mRNA levels of type 1 arginase (Arg1), Ym1 and FIZZ1, the markers of M2 macrophages, ${ }^{13,16-18}$ expressed by conjunctival tissue in this EAC model of WT-BALB/c mice (WT-EAC), when compared with PBS-control mice (Fig. 1a). The increased gene expression of these M2 markers was also detected in the ocular surface draining cervical lymph nodes (CLNs) of WT-EAC mice (Fig. 1b).

To confirm that the upregulated mRNA levels of M2 markers represent the macrophage polarization in the murine EAC model, immunofluorescent double staining was performed using antibodies against Arg1, Ym1, or FIZZ1, together with F4/80 antibody, the major cell surface marker for macrophages. ${ }^{19}$ As shown in Fig. 1C, the significant increase of macrophages, positively stained with $\mathrm{F} 4 / 80\left(\mathrm{~F} 4 / 80^{+}\right)$, was detected at the ocular surface, mainly in the conjunctiva and stromal area of the WT-EAC mice when compared with PBS controls. Among the $\mathrm{F} 4 / 80^{+}$macrophages, some cells also displayed positive immunoreactivities to $M 2$ macrophage markers, Arg1, Ym1, and FIZZ1, as measured percentages of double-positive cells with colocalization of each M2 marker and F4/80 (Fig. 1e). This phenotype of M2 macrophages was also clearly observed in the CLNs. Compared with PBS controls, the $\mathrm{F} 4 / 80^{+}$macrophages largely increased in the $\mathrm{CLNs}$ of WT-EAC mice, and most macrophages co-stained with the M2 markers, Arg1, Ym1, and FIZZ1 (Fig. 1d). The percentages of double-positive cells with colocalization of macrophage marker F4/80 and each M2 marker in conjunctiva and CLNs were quantitatively measured in these mice, as shown in Fig. 1e. We further calculate the colocalization percentage using M2 markers as denominator. The results show that about $50-60 \%$ of macrophages are related to the total population of Arg1, Ym1, and FIZZ1 producers in WT-EAC model (Fig. S2 in Supplementary Information).

M2 Macrophages were identified to express TSLPR and produce Th2-inducing cytokine OX40L

It is well known that pro-allergic cytokine TSLP can activate dendritic cells to produce OX40L, a Th2-inducing cytokine 
a

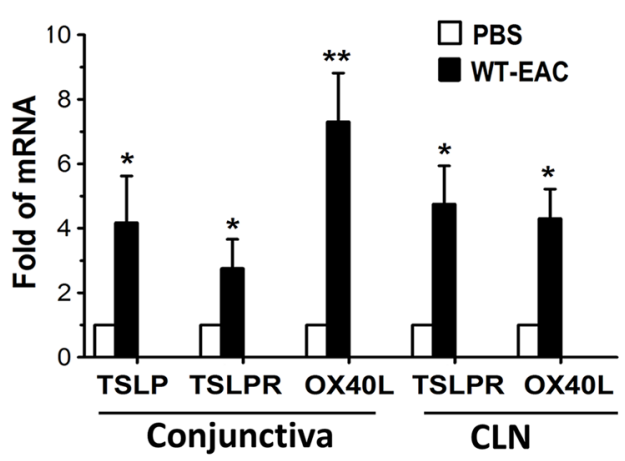

d
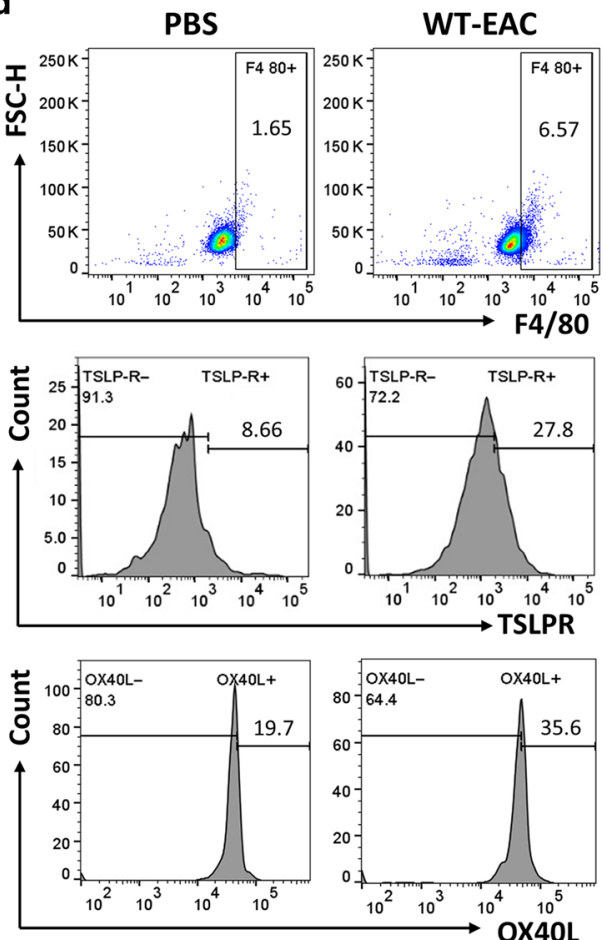

b
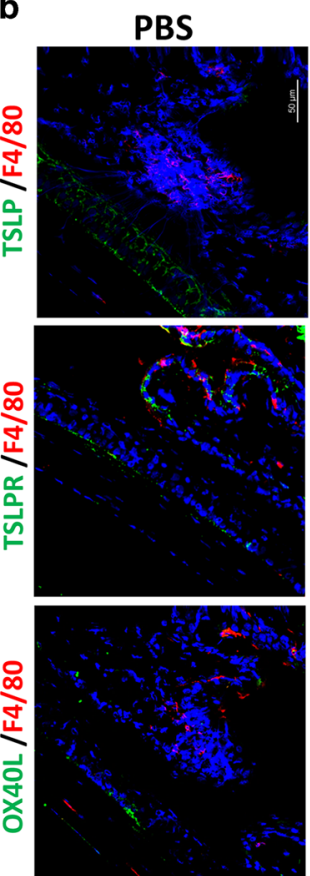

c
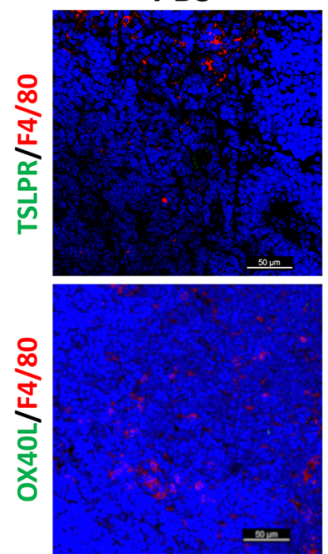

WT-EAC
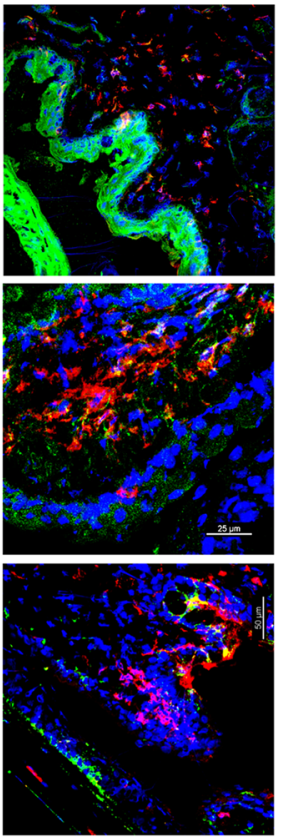

WT-EAC
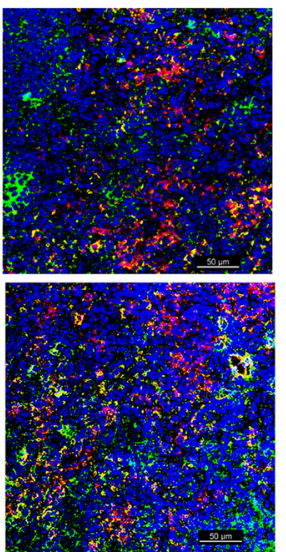

Fig. 2 M2 Macrophages were shown to express TSLPR and produce Th2-inducing cytokine OX40L. a mRNA expression of TSLP, TSLPR, and OX40L in conjunctiva and CLNs of PBS and WT-EAC mice. b Representative images of immunofluorescent double staining of TSLP, TSLPR, and OX40L, together with F4/80 at ocular surface, and in CLNs (c) of PBS, WT-EAC and TSLPR ${ }^{-1-}$-EAC mice. d Flow cytometry showed the expression levels of TSLPR and OX40L by macrophages from CLN cells of PBS and WT-EAC mice. Results shown are the Mean \pm SD. ${ }^{*} P<0.05$, ${ }^{* * P}<0.01 ; n=3$, compared with controls

that stimulates Th2-dominant allergic inflammatory hyper response. ${ }^{3,4,20,21}$ A new study has reported that macrophages could respond to TSLP. ${ }^{13}$ However, it is not well documented whether macrophages express TSLPR and OX40L in response to epithelial TSLP. It is unknown how M2 macrophages were primed by a pollen allergen in allergic disease. The TSLP signaling molecules were evaluated at mRNA and protein levels using RTqPCR, immunofluorescent double staining and flow cytometric assays. The transcripts of TSLP, TSLPR, and OX40L were found to be significantly upregulated by conjunctival cells, and the expression levels of TSLPR and OX40L also increased in CLNs of WT-EAC mice compared with PBS-control mice (Fig. 2a).

Immunofluorescent staining revealed that $\mathrm{F} 4 / 80^{+}$macrophages were largely accumulated in the conjunctival stroma area of WTEAC mice while only a few macrophages were present in the ocular surface of control mice. As shown in Fig. 2b, Immunofluorescent double staining showed that TSLP mainly expressed by epithelial cells of cornea and conjunctiva, while
$\mathrm{F} 4 / 80^{+}$macrophages were present in conjunctival stroma side. Among them, some were co-stained with TSLPR and/or OX40L, suggesting these immunoreactivities may be generated, at least partly, by macrophages. The $\mathrm{F} 4 / 80^{+}$macrophages were also observed to be co-stained with TSLPR and OX40L in CLNs (Fig. 2c).

Using freshly isolated lymph cells from CLNs in mouse model, flow cytometric analysis confirmed that the population of macrophages increased about fourfold in EAC mice compared with PBS-control mice. The percentages of $\mathrm{TSLPR}^{+}$macrophages increased more than threefold, and $\mathrm{OX}_{40 \mathrm{~L}^{+}}$macrophages increased near twofold in EAC model (Fig. 2d). The negative controls consisted of fluorescence minus one (FMO) cells in flow cytometry for Fig. $2 d$ were provided in Supplementary data Fig S1.

M2 macrophages produce Th2-attracting chemokines TARC and MDC

Thymus and activation-regulated chemokine (TARC/CCL17) and macrophage-derived chemokine (MDC/CCL22) are mainly 

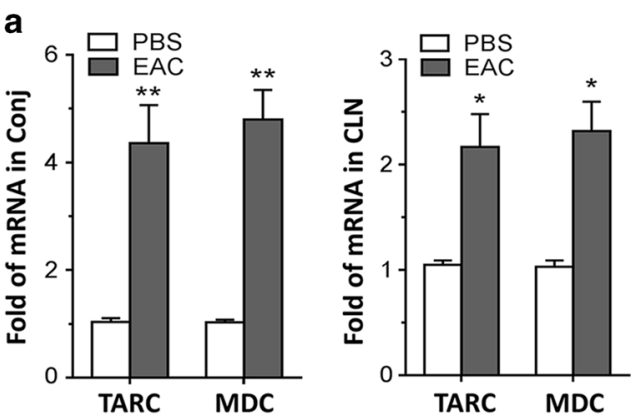

b
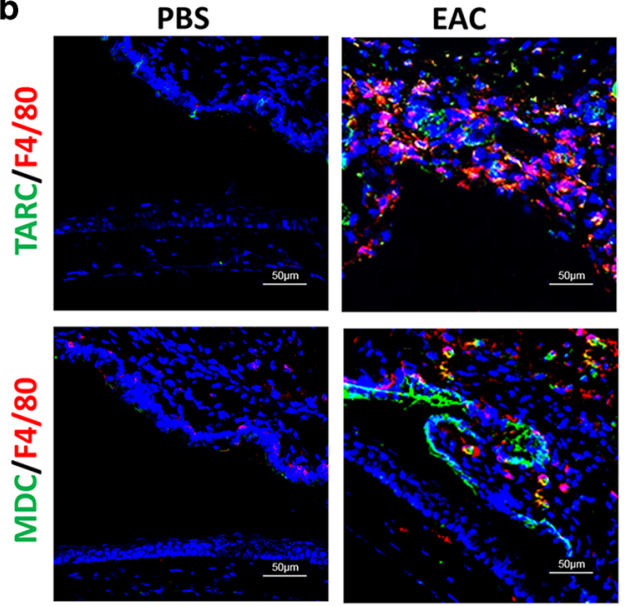

C
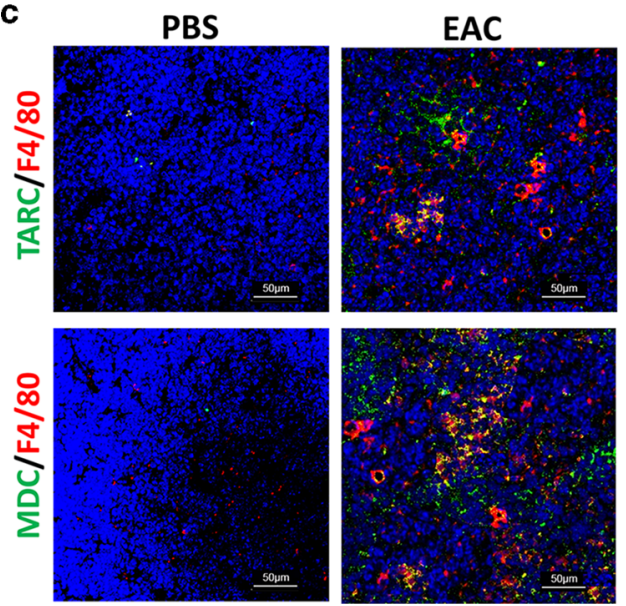

Fig. $3 \mathrm{M} 2$ macrophages produce Th2-attracting chemokines TARC and MDC. a mRNA expression of TARC and MDC by conjunctiva (Conj) and CLNs in PBS control and WT-EAC mice. b Representative images of immunofluorescent double staining of TARC and MDC, together with F4/80 antibody at ocular surface, and in CLNs (c) of PBS and WT-EAC mice. Results shown are the Mean \pm SD. ${ }^{*} P<0.05$, ${ }^{*} P<0.01 ; n=5$, compared with controls

produced by macrophages and dendritic cells, as well as epithelial cells. TARC and MDC are Th2-attracting chemokines, and play an important role in recruiting Th2 cells into the inflammatory sites and regulating Th2-related immune responses in allergic diseases. $^{22-24}$ TARC and MDC were found to be upregulated at mRNA and protein levels in EAC model. The transcripts of TARC and MDC increased $4.4 \pm 0.7$ and $4.8 \pm 0.6$ fold, respectively in conjunctiva, and $2.2 \pm 0.3$ and $2.6 \pm 0.3$ fold, respectively in CLNs (Fig. 3a). The stimulated production of TARC and MDC by M2 macrophages was confirmed at protein level. Immunofluorescent double staining showed some $\mathrm{F} 4 / 80^{+}$macrophages co-stained with TARC or MDC antibody and appeared as orange-colored cells in the ocular surface (Fig. 3b) and CLNs (Fig. 3c).

TSLP/TSLPR/OX40L signaling was involved in M2 macrophage polarization in EAC model

Compared with WT-EAC mice, the allergic signs on ocular surface (Fig. 4a), and stimulated Th2-dominant inflammatory response by ocular mucosa, especially conjunctival tissues, were dramatically reduced or eliminated in $\mathrm{BALB} / \mathrm{c}$ based $T S L P R^{-1-}$ mice that were treated with SRW at same way to induce EAC model. As shown in Fig. 4b, the mRNA levels of Th2 cytokines (IL-4, IL-5, and IL-13) were significantly upregulated in conjunctival tissue from WT-EAC, but not in those from $T S L P R^{-/-}$-EAC mice. The immunohistochemical staining confirmed these results at protein levels. As shown in Fig. 4c, the increased immunoreactivities of IL-4, IL-5, and IL-13 by Th2 cells at ocular surface mucosa was only seen in WT-EAC but not in TSLPR ${ }^{-/-}$-EAC mice.

We then investigated the M2 macrophage polarization in $T S L P R^{-1-}$ mice treated by SRW for EAC model. As shown in Fig. 1a, the mRNA levels of three M2 markers, Arg1, Ym1, and FIZZ1, by conjunctiva were markedly induced in WT-EAC mice, but not in $T S L P R^{-/-}$mice.

The similar pattern was observed in CLNs (Fig. 1b). Double staining also showed that the numerous $\mathrm{F} 4 / 80^{+}$macrophages co-stained with Arg1, Ym1 or FIZZ1 were only observed at the ocular surface conjunctival tissues (Fig. 1c) and CLNs (Fig. 1d) in WT-EAC but not in TSLPR ${ }^{-1-}$-EAC mice. These findings suggest that TSLP/TSLPR/OX40L signaling was involved in the SRW pollen-induced allergic inflammation as well as M2 macrophage polarization.

Pollen/TSLP/TSLPR plays a role in priming M2 Macrophages that produce OX40L, TARC, and MDC in BM-derived macrophages

To further confirm the role of pollen in priming $M 2$ macrophage polarization via TSLP/TSLPR signaling, BM-derived macrophages were generated from wild-type BALB/c mice. Flow cytometric analysis identified that the more than $92 \%$ of BM-derived cells were positive to macrophage cell surface markers F4/80 and CD11b (Fig. 5a). About $11 \%$ of these $\mathrm{F} 4 / 80^{+}$macrophages were found to express TSLPR. The TSLPR ${ }^{+}$macrophages increased 3.6-fold when treated with $50 \mu \mathrm{g}$ of SRWe (aqueous extract of defatted SRW pollen), and fourfold by $10 \mathrm{ng}$ of TSLP stimulation (Fig. 5b). The OX40L, a downstream molecule in response to TSLP, was also found to be produced by about $9 \%$ of BM-macrophages, and was stimulated to 3-fold and 1.8-fold, respectively, by SRWe and TSLP (Fig. 5c). Immunofluorescent staining confirmed this stimulating responses of TSLPR and OX40L by BM-macrophages when exposed to SRWe and TSLP (Fig. 5d). Their stimulated production of OX40L, TARC, and MDC at protein levels were quantitatively confirmed by ELISA (Fig. 5e).

When compared BM-macrophages derived from WT and $T S L P R^{-1-}$ mice, we observed that SRWe significantly upregulated the mRNA expression of M2 markers, Arg1, Ym1, and FIZZ1, by BM-macrophages from WT mice, but not by those from TSLPR ${ }^{-1-}$ mice (Fig. 6a). Immunofluorescent staining confirmed the findings at protein levels. As shown in Fig. 6b, SRWe increased the immunoreactivities of Arg1, Ym1, and FIZZ1 significantly in the macrophages derived from WT mice, but not from $T S L P R^{-/-}$mice. These results indicate that SRW pollen promotes the polarization of M2 macrophages via TSLP/TSLPR signaling pathway.

\section{DISCUSSION}

The cellular and molecular mechanisms by which pollen allergen triggers allergic inflammation have not been well elucidated. The knowledge gaps prevent us from developing new targeted therapies to cure allergic diseases. Recent advances in the field 

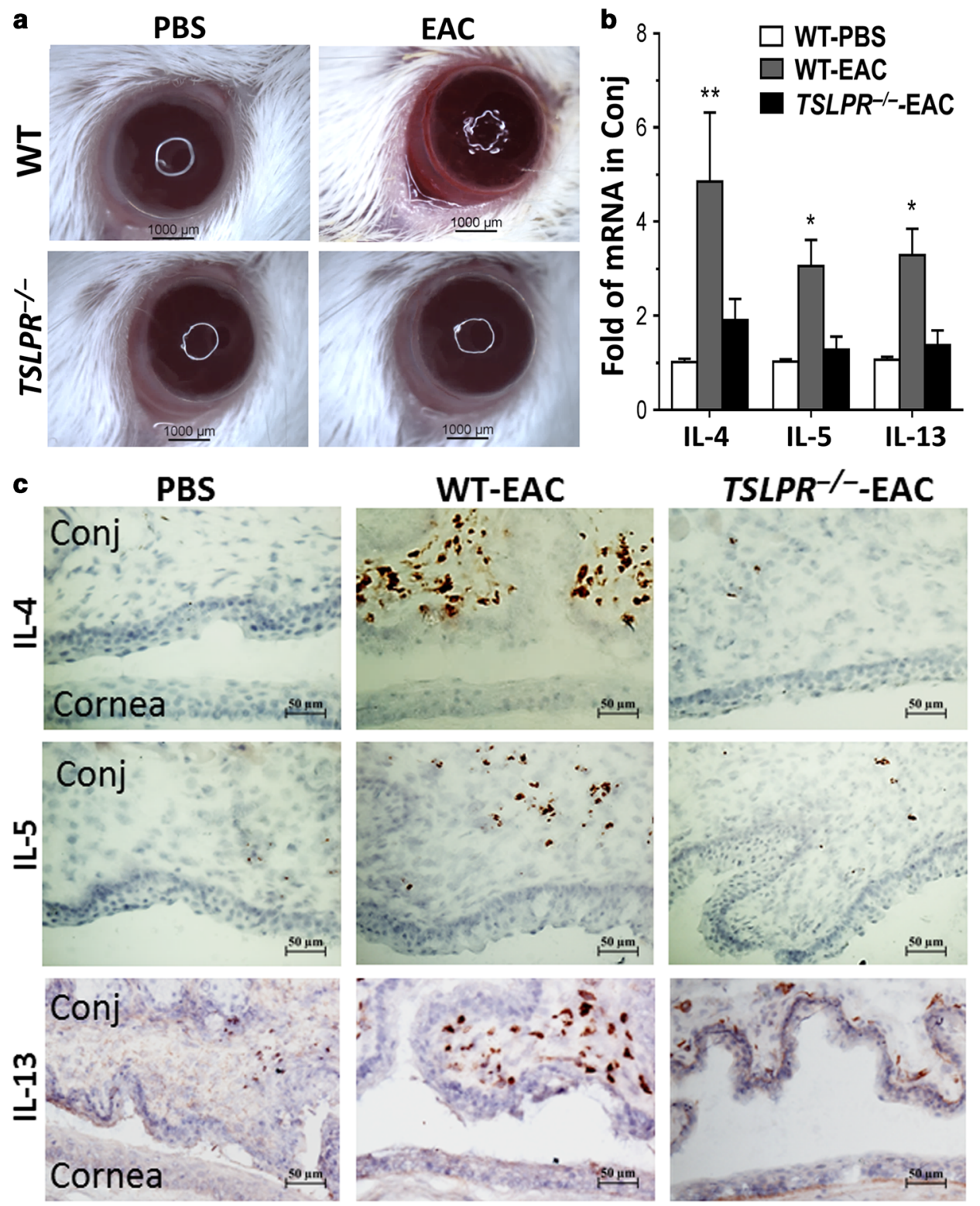

Fig. 4 The clinical signs and the Th2 cytokine levels in PBS control, WT-EAC and TSLPR ${ }^{-1}-$-EAC mice. a The typical manifestations of allergic conjunctivitis, including lid edema, conjunctival redness, chemosis, tearing, and frequent scratching of the eye lids, were observed in WT-EAC mice, but not in TSLPR ${ }^{-1-}$ - EAC mice. b mRNA expression of IL-4, IL-5, and IL-13 by conjunctiva (Conj) and CLNs in PBS control, WT-EAC and $T S L P R^{-1-}$-EAC mice. c Representative images of immunohistochemical staining of IL-4, IL-5, and IL-13 at ocular surface of PBS, WT-EAC, and $T S L P R^{-1-}$-EAC mice. Results shown are the Mean \pm SD. ${ }^{*} P<0.05,{ }^{* *} P<0.01 ; n=5$, compared with controls

demonstrate that epithelium-derived pro-allergic cytokine TSLP plays a key role in initiating allergic inflammation in asthma, atopic dermatitis and ocular allergy. Dendritic cells have been focused to be one major cell type that possesses TSLPR and responds to TSLP to produce $\mathrm{OX} 40 \mathrm{~L}$, a Th2-inducing cytokine that triggers Th2dominent allergic inflammation..$^{3,4,8,25}$ Macrophages are also major innate immune cells with well-established roles in regulating the adaptive immune responses and inflammation. ${ }^{26,27}$ However, their contribution to pollen-triggered allergic inflammation has not been elucidated. This study revealed that SRW Pollen promotes M2 macrophage polarization via TSLP/TSLPR signaling to amplify the allergic inflammation.

M2 macrophage polarization has been reported in allergic diseases including asthma, dermatitis, etc., ${ }^{10,12,13}$ but not yet in ocular allergy, nor in pollen-induced allergy. Using a murine model of EAC induced by SRW pollen, the M2 macrophage polarization was observed in conjunctival tissue and ocular surface draining CLNs of wild-type BALB/c mice, as evaluated at mRNA and protein levels of three M2 markers, Arg1, Ym1 and FIZZ1, by RT-qPCR and immunofluorescent staining (Fig. 1).

Although TSLP has been reported to amplify the M2 macrophage differentiation, ${ }^{13}$ more evidence is needed to confirm whether macrophages possess TSLPR and produce OX40L in response to TSLP. As evaluated by RT-qPCR, immunofluorescent double staining and flow cytometry, TSLPR was detected by macrophages of untreated mice, and its expression significantly increased by $\mathrm{M} 2$ macrophages in conjunctiva and CLNs of EAC model, accompanied by stimulated OX40L (Fig. 2). The findings suggest that like dendritic cells, macrophages express TSLPR, are able to respond to TSLP, and produce OX40L that stimulate Th2 cytokine production in pollen-induced allergic conditions. In addition to TSLPR and OX40L, M2 macrophages were also shown 


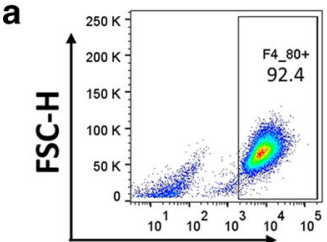

$\mathrm{F} 4 / 80$

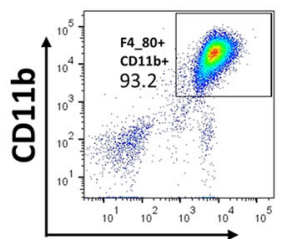

$\mathrm{F} 4 / 80$ b

UT
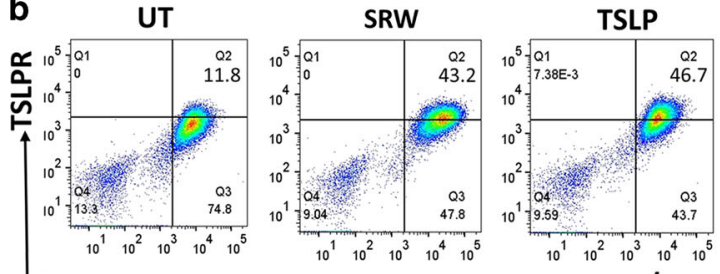

$\rightarrow$ F4/80
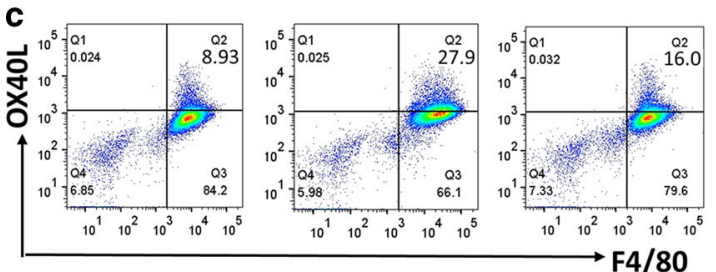

d
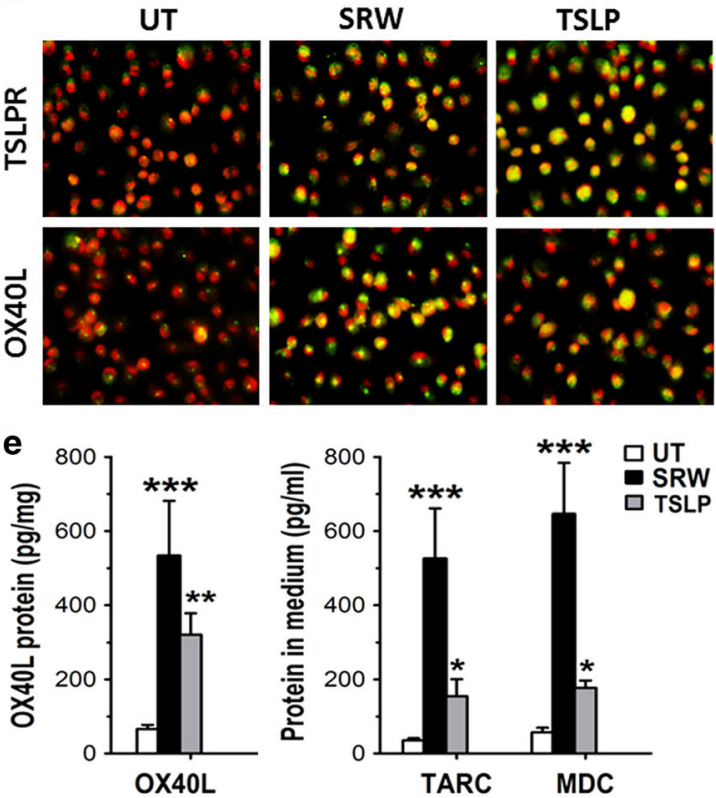

Fig. 5 BM-derived macrophages express TSLPR and produce OX40L, TARC, and MDC when exposed to SRWe and TSLP. a Flow cytometry showed the purity of BM-macrophages by markers CD11b and F4/80. b, c Flow cytometry showed the percentages of TSLP ${ }^{+}$and OX40L $\mathrm{O}^{+} \mathrm{cells}_{\text {, }}$ respectively, in macrophages when exposed to SRWe and TSLP. d Representative images of immunofluorescent staining of TSLPR and OX40L (green) in macrophages by SRWe and TSLP with propidium iodide (PI, red) as nuclear counterstaining. e Quantitative protein levels of OX40L, TARC and MDC in macrophages by ELISA. Results shown are the Mean \pm SD. ${ }^{*} P<0.05,{ }^{* *} P<0.01$ and ${ }^{* * *} P<0.001 ; n=5$, compared with controls

to express and secrete higher levels of TARC and MDC in ocular surface conjunctiva and the draining CLNs (Fig. 3), the major Th2attracting chemokines associated with allergic sensitization, inflammatory response, and severity. $23,28,29$

To confirm that pollen promotes M2 phenotype of macrophages via TSLP/TSLPR signaling in allergic inflammation, $T S L P R^{-/-}$mice were used for study. TSLPR ${ }^{-/-}$mice have been known to block allergic inflammation in asthma and dermatitis, ${ }^{13,30,31}$ but not yet in ocular allergy. Using in vivo murine EAC model induced by SRW pollen, $T S L P R^{-1-}$ mice failed to respond to SRW pollen challenge. TSLPR ${ }^{-1-}$-EAC mice did not show typical ocular manifestations of allergic conjunctivitis (Fig. 4a) and Th2 cytokine hyperresponse at both mRNA and protein levels (Fig. 4b, c). These mice did not show any increased infiltration of macrophages with $\mathrm{M} 2$ markers (Arg1, Ym1, and FIZZ1) in conjunctiva and CLNs, which were observed only in WT-EAC mice (Fig. 1a-d).

It has been well documented that Th2 cytokines IL-13 and IL-4 induce M2 macrophage while Th1 cytokine IFNY and LPS promote M1 phenotype. ${ }^{10,13,32,33}$ The M2 macrophages-produced OX40L participates in promoting Th2 cytokines production. With lower levels of Th1 cytokine IFN $\gamma$ in the EAC mice as we recently reported, ${ }^{15}$ the micro-environmental condition favors macrophage polarization towards to $\mathrm{M} 2$ phenotype in the SRWinduced EAC model.

Using in vitro cultures of bone marrow-derived macrophages, direct responses to SRW pollen and TSLP were evaluated. Murine BM-macrophages were about $92 \%$ pure based on percentages of positive cells to $\mathrm{CD} 11 \mathrm{~b}$ and $\mathrm{F} 4 / 80$ by flow cytometric assay (Fig. 5a). About $12 \%$ of BM-macrophages expressed TSLPR in untreated cells, The TSLPR ${ }^{+}$BM-macrophages increased to 3.6and 4-fold respectively when exposed to SRWe and TSLP (Fig. 5b). About $9 \%$ of BM-macrophages expressed OX40L in untreated cells, the $\mathrm{OX}_{40 \mathrm{OL}}{ }^{+}$cells increased to 3.1 - and 1.8-fold respectively when challenged by SRWe and TSLP (Fig. 5c). SRWe and TSLP also stimulated the production of OX40L and secretion of TARC and MDC (Fig. 5d, e). The baseline expression and response patterns of Th2-inducing TSLPR and OX40L, and Th2-atracting chemokines TARC and MDC in vitro culture model of BM-macrophages are similar to those in vivo EAC model. These results indicate that macrophages possess TSLPR and are able to response to TSLP just similar to dendritic cells.

By comparing BM-macrophages from WT and TSLPR ${ }^{-1-}$ mice, we further confirmed that SRWe significantly stimulated mRNA expression and protein production of $\mathrm{M} 2$ markers Arg1, Ym1, and FIZZ1 in the cells only from WT mice but not from $T S L P R^{-1-}$ mice (Fig. 6). Taken together with strong evidence from in vivo EAC models of WT and $T S L P R^{-/-}$mice, all findings demonstrate that SRW pollen prime macrophages towards to alternative activated phenotype via TSLP/TSLPR/OX40L signaling.

In conclusion, our findings reveal that macrophages play important role in Th2-dominent allergic inflammation by polarization to $\mathrm{M} 2$ phenotype in SRW pollen-induced EAC murine model. Like dendritic cells, M2 macrophages possess TSLPR and can respond to proallergic cytokine TSLP to produce OX40L, and M2 macrophages also produce Th2-attracting chemokines, TARC and MDC, which further promote Th2 cells to amplify allergic inflammation.

\section{METHODS}

Animals

The animal protocol for this study was approved by the Institutional Animal Care and Use Committee (IACUC), Center for Comparative Medicine, Baylor College of Medicine. All animals used in this study were maintained in specific pathogen-free conditions in microisolator cages and were treated in accordance with the National Institutes of Health guide for the care and use of 
a

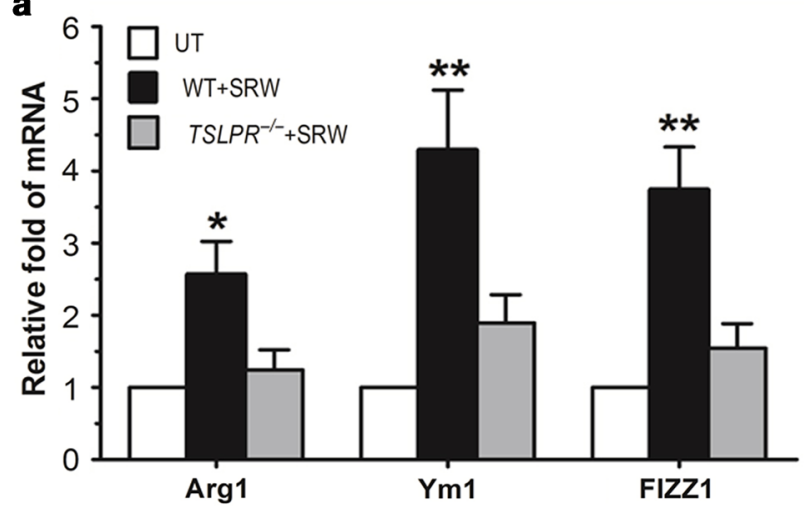

b
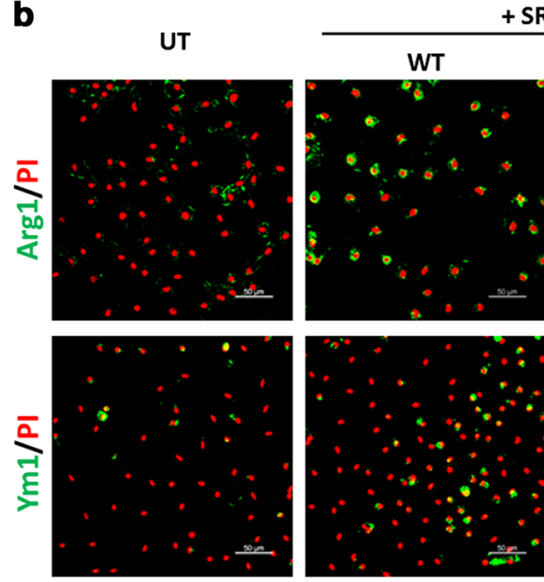

+ SRW
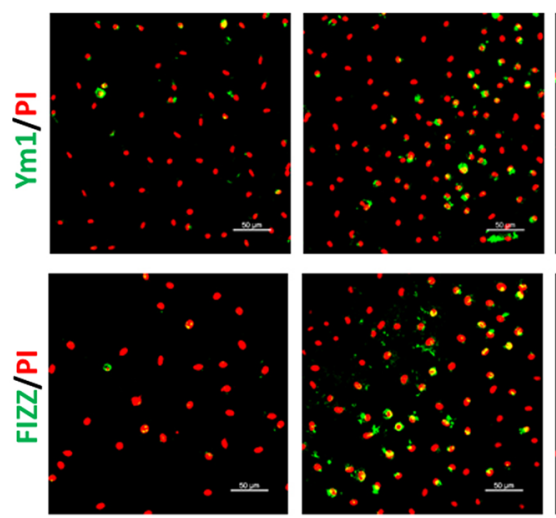

Fig. 6 M2 markers, Arg1, Ym1, and FIZZ1, were stimulated by SRWe in BM-macrophages from WT mice, but not from $T S L P R^{-1-}$ mice. a mRNA expression of Arg1, Ym1, and FIZZ1 in BM-macrophages derived WT and TSLPR ${ }^{-/-}$mice treated with SRWe. b Representative images of immunofluorescent staining of Arg1, Ym1, and FIZZ1 in macrophages exposed to SRWe. Results shown are the Mean \pm SD. ${ }^{*} P<0.05,{ }^{* *} P<0.01 ; n=5$, compared with controls

Laboratory animals (NIH Publications No. 8023, revised 1978). Wild-type BALB/c female mice aged at 6-8 weeks were purchased from the Jackson Laboratory (Bar Harbor, ME). The homozygous TSLPR knockout $\left(T_{S L P R^{-1}}\right.$ ) mice in BALB/c background ${ }^{30}$ were kindly provided by Professor Steven F. Ziegler (Benaroya Research Institute and Department of Immunology, University of Washington School of Medicine).

Murine EAC model induced by SRW pollen

The mouse EAC model was induced using wild-type BALB/c and TSLPR ${ }^{-1-}$ mice with previously reported methods. ${ }^{7,8,15,34,35}$ In brief, mice were immunized with $50 \mathrm{\mu g}$ of SRW pollen (Greer Lab, Lenoir, NC) by footpad injection on day 0 for sensitization. EAC was induced by giving topical applications of $1.5 \mathrm{mg}$ SRW pollen into each eye once at days 10-15. Untreated normal mice and PBS eyedrop treated SRW-sensitized mice were used as controls. Animals were examined clinically for signs, including chemosis, conjunctival redness, lid edema, and tearing. The conjunctiva, CLNs and whole eye balls were collected for studies. Six mice per

group were used in each experiment, and each experiment was repeated 3-5 times.

In vitro responses of mouse bone marrow-derived macrophages to aqueous extract of defatted SRW pollen (SRWe) and TSLP

Macrophages from wild-type and TSLPR ${ }^{-1-}$ mice were generated from mouse bone marrow as previously described ${ }^{36,37}$ with modification. Briefly, bone marrow cells isolated from femurs and tibiae were cultured in RPMI- 1640 with $10 \%$ FBS containing $20 \mathrm{ng} / \mathrm{ml}$ of $\mathrm{rmM}-\mathrm{CSF}$. On days 7-8, the adherent macrophages were harvested with trypsin/EDTA for experiments. The macrophages at $1-5 \times 10^{6} /$ well in 12 - or 6 -well plates were incubated for $4-24 \mathrm{~h}$ in medium alone, or treated with SRWe $(50 \mu \mathrm{g} / \mathrm{ml})$ or rmTSLP $(10 \mathrm{ng} / \mathrm{ml})$ for mRNA and flow cytometric assays. The macrophages at $1 \times 10^{5} /$ chamber in 8-chamber slides were incubated for $24 \mathrm{~h}$ with different treatments for immunofluorescent staining.

Flow cytometric analysis for the isolated CLN cells from mouse models and in vitro BM-macrophage cultures

Flow cytometry was performed as previous reported methods. ${ }^{38-40}$ In brief, single-cell suspensions from fresh mouse CLNs or cultured BM-macrophages were filtered by a $70-\mu \mathrm{m}$ cell strainer, centrifuged, and resuspended. Cells were incubated with a stain kit (LIVE/DEAD Fixable Yellow Dead Cell Stain Kit, L34959, Thermo Fisher Scientific, Waltham, MA), followed by staining with antiCD11b-APC (clone M1/70; eBioscience, San Diego, CA), anti-F4/80APC/Cy7 (clone BM8; Biolegend), anti-TSLPR-PE (FAB5461P; R\&D Systems, Minneapolis, MN), and anti-OX40L-PE/Cy7 (clone RM134L; Biolegend).

The gating strategy used in this study was as follows: dead cells were excluded by gating live dye negative cells, subsequently gated on the basis of forward scatter height versus forward scatter area (singlets 1), then gated on side scatter height versus side scatter area (singlets 2). Cells were then gated on $\mathrm{F} 4 / 80^{+} \mathrm{CD} 11 \mathrm{~b}^{+}$ cells, and frequencies of $\mathrm{F} 4 / 80^{+} \mathrm{TSLPR}^{+}$and $\mathrm{F} 4 / 80^{+} \mathrm{OX}_{40 \mathrm{~L}}{ }^{+}$were recorded. Negative controls consisted of FMO cells. A BD Canto II Benchtop cytometer (Becton Dickinson, San Jose, CA) was used for flow cytometry, and data were analyzed with BD Diva software v6.7 (BD Biosciences, San Jose, CA) and FlowJo software v10. (Tree Star Inc., Ashland, OR).

Total RNA extraction, reverse transcription (RT), and quantitative real-time PCR (qPCR)

As previously reported methods, ${ }^{7,8,41}$ total RNA was extracted with RNeasy Plus Mini Kit (Qiagen, Valencia, CA) according to the manufacturer's instructions, quantified with a spectrophotometer (NanoDrop ND-2000; Thermo Scientific, Wilmington, DE), and stored at $-80^{\circ} \mathrm{C}$ before use. The first strand cDNA was synthesized by RT from $1.0 \mu \mathrm{g}$ of total RNA using Ready-To-Go You-Prime FirstStrand Beads as previously described. qPCR was performed in StepOnePlus ${ }^{\text {TM }}$ Real-Time PCR System (Applied Biosystems, Foster City, CA), and TaqMan gene expression assays used for this study were listed in Table 1 . The results were analyzed by the comparative threshold cycle method and normalized by GAPDH as an internal control. The mRNA fold changes in EAC model were normalized by the average of mRNA expression levels in the tissues of PBS-control mice. So the fold for control always equal to 1 without error bar.

Enzyme-linked immunosorbent assay (ELISA)

Double-sandwich ELISA kits for mouse OX40L, TARC, and MDC were from BioLegend (San Diego, CA), and performed according to manufacturers' protocols similar to our previous reports. ${ }^{21,42}$ Absorbance was read at $450 \mathrm{~nm}$ with a reference wavelength of $570 \mathrm{~nm}$ by Infinite M200 microplate reader (Tecan US, Inc., Morrisville, NC). 
Table 1. TaqMan gene expression assays used for RT-qPCR

\begin{tabular}{ll}
\hline TaqMan gene & Assay ID \\
\hline GAPDH & Mm99999915_g1 \\
Arg1 & Mm00475988_m1 \\
Ym1 & Mm00657889_mH \\
FIZZ1 & Mm00445109_m1 \\
TSLP & Mm00498739_m1 \\
TSLPR & Mm00497362_m1 \\
OX40L & Mm00437214_m1 \\
TARC & Mm00516136_m1 \\
MDC & Mm00436439_m1 \\
IL-4 & Mm00445259_m1 \\
IL-5 & Mm99999063_m1 \\
IL-13 & Mm00434204_m1
\end{tabular}

Table 2. Antibodies used for immunohistochemical and immunofluorescent staining

\begin{tabular}{llll}
\hline Antibodies & Company & Cat.\# & Dilution \\
\hline Arg1 & Abcam & Ab60176 & $1: 50$ \\
Ym1 & Abcam & Ab93034 & $1: 100$ \\
FIZZ1 & Abcam & Ab39626 & $1: 100$ \\
F4/80 & Biolegend & B226028 & $1: 100$ \\
TSLP & ProSci & 4023 & $1: 200$ \\
TSLPR & Prosci & 4209 & $1: 100$ \\
OX40L & Santa Cruz & SC-10952 & $1: 100$ \\
TARC & Bioss & BS-2453R & $1: 200$ \\
MDC & Bioss & BS-1761R & $1: 200$ \\
IL-4 & BioLegend & 504101 & $1: 100$ \\
IL-5 & BioLegend & 504301 & $1: 100$ \\
IL-13 & Santa Cruz & SC-1776 & $1: 100$ \\
\hline
\end{tabular}

Immunohistochemical and immunofluorescent staining

Cryosections at $8 \mu \mathrm{m}$ of mouse eyeballs and CLNs were cut with a cryostat (HM 500; Micron, Waldorf, Germany), and stored at $-80^{\circ} \mathrm{C}$. Immunohistochemical and immunofluorescent staining were performed as previously described. ${ }^{7,8,41}$ Primary antibodies used for this study were listed in Table 2. Secondary antibody alone or isotype IgG were used as negative controls. The results were photographed with an epifluorescence microscope (Eclipse 400; Nikon, Garden City, NY) using a digital camera (DMX 1200; Nikon).

Statistical analysis

Student's $t$-test was used to compare differences between two groups. One-way ANOVA test was used to make comparisons among three or more groups, followed by Dunnett's post hoc test. $P$ values $<0.05$ were considered statistically significant.

\section{ACKNOWLEDGEMENTS}

The authors thank Professor Steven F. Ziegler (Benaroya Research Institute and Department of Immunology, University of Washington School of Medicine) for great support by providing the TSLPR knockout mice. This work was partially supported by National Institutes of Health grant EY023598 (DQL), Core Grant for Vision Research EY002520, an unrestricted grant from Research to Prevent Blindness, LQ17H120010, Y20160056, the Oshman Foundation and the William Stamps Farish Fund.

\section{AUTHOR CONTRIBUTIONS}

D-Q.L., S.C.P. and F.L. designed the research; R.D., X.C., Y.Z., F.B., N.G., J.H. and R.G.dP. performed the experiments; R.D., X.C., Y.Z., F.B. and D-Q.L. analyzed the data and prepared figures; R.D., X.C. and D-Q.L. wrote the manuscript; S.C.P. performed English editing; and all authors reviewed the manuscript.

\section{ADDITIONAL INFORMATION}

The online version of this article (https://doi.org/10.1038/s41385-019-0187-8) contains supplementary material, which is available to authorized users.

Competing interests: The authors declare no competing interests.

Publisher's note: Springer Nature remains neutral with regard to jurisdictional claims in published maps and institutional affiliations.

\section{REFERENCES}

1. Kato, A. \& Schleimer, R. P. Beyond inflammation: airway epithelial cells are at the interface of innate and adaptive immunity. Curr. Opin. Immunol. 19, 711-720 (2007).

2. Ueta, M. \& Kinoshita, S. Ocular surface inflammation mediated by innate immunity. Eye Contact Lens. 36, 269-281 (2010).

3. Liu, Y. J. Thymic stromal lymphopoietin and OX40 ligand pathway in the initiation of dendritic cell-mediated allergic inflammation. J. Allergy Clin. Immunol. 120, 238-244 (2007)

4. Ziegler, S. F. \& Liu, Y. J. Thymic stromal lymphopoietin in normal and pathogenic T cell development and function. Nat. Immunol. 7, 709-714 (2006).

5. Guo, Z. et al. IL-33 promotes airway remodeling and is a marker of asthma disease severity. J. Asthma 51, 863-869 (2014).

6. Haenuki, Y. et al. A critical role of IL-33 in experimental allergic rhinitis. J. Allergy Clin. Immunol. 130, 184-194 e111 (2012).

7. Li, J. et al. Pollen/TLR4 innate immunity signaling initiates IL-33/ST2/Th2 pathways in allergic inflammation. Sci. Rep. 6, 36150 (2016).

8. Li, D. Q. et al. Short ragweed pollen triggers allergic inflammation through Toll-like receptor 4-dependent thymic stromal lymphopoietin/OX40 ligand/ OX40 signaling pathways. J. Allergy Clin. Immunol. 128, 1318-1325 e1312 (2011).

9. Zheng, X. et al. TSLP and downstream molecules in experimental mouse allergic conjunctivitis. Invest. Ophthalmol. Vis. Sci. 51, 3076-3082 (2010).

10. Saradna, A., Do, D. C., Kumar, S., Fu, Q. L. \& Gao, P. Macrophage polarization and allergic asthma. Transl. Res. 191, 1-14 (2018).

11. Robbe, P. et al. Distinct macrophage phenotypes in allergic and nonallergic lung inflammation. Am. J. Physiol.-Lung C. 308, L358-L367 (2015).

12. Suzuki, K., Meguro, K., Nakagomi, D. \& Nakajima, H. Roles of alternatively activated M2 macrophages in allergic contact dermatitis. Allergol. Int. 66, 392-397 (2017).

13. Han, H. et al. Thymic stromal lymphopoietin amplifies the differentiation of alternatively activated macrophages. J. Immunol. 190, 904-912 (2013).

14. Kurowska-Stolarska, M. et al. IL-33 amplifies the polarization of alternatively activated macrophages that contribute to airway inflammation. J. Immunol. 183, 6469-6477 (2009)

15. Chen, X. et al. IL-27 signaling deficiency develops Th17-enhanced Th2-dominant inflammation in murine allergic conjunctivitis model. Allergy, https://doi.org/ 10.1111/all.13691 (2018).

16. Moreira, A. P. et al. Serum amyloid $P$ attenuates $M 2$ macrophage activation and protects against fungal spore-induced allergic airway disease. J. Allergy Clin. Immunol. 126, 712-721 (2010).

17. Zhao, Q. et al. 2-Deoxy-d-glucose treatment decreases anti-inflammatory $M 2$ macrophage polarization in mice with tumor and allergic airway inflammation. Front. Immunol. 8, 637 (2017).

18. $\mathrm{Nie}, \mathrm{H}$. et al. Phenotypic switch in lung interstitial macrophage polarization in an ovalbumin-induced mouse model of asthma. Exp. Ther. Med. 14, 1284-1292 (2017).

19. Eske, K., Breitbach, K., Kohler, J., Wongprompitak, P. \& Steinmetz, I. Generation of murine bone marrow derived macrophages in a standardised serum-free cell culture system. J. Immunol. Methods 342, 13-19 (2009).

20. Ito, T. et al. TSLP-activated dendritic cells induce an inflammatory T helper type 2 cell response through OX40 ligand. J. Exp. Med. 202, 1213-1223 (2005).

21. Deng, R. et al. A potential link between bacterial pathogens and allergic conjunctivitis by dendritic cells. Exp. Eye Res. 120, 118-126 (2014).

22. Scheu, S., Ali, S., Ruland, C., Arolt, V. \& Alferink, J. The C-C chemokines CCL17 and CCL22 and their receptor CCR4 in CNS autoimmunity. Int. J. Mol. Sci. 18, https:// doi.org/10.3390/ijms18112306 (2017). 
23. Bisset, L. R. \& Schmid-Grendelmeier, P. Chemokines and their receptors in the pathogenesis of allergic asthma: progress and perspective. Curr. Opin. Pulm. Med. 11, 35-42 (2005).

24. Horikawa, T. et al. IFN-gamma-inducible expression of thymus and activationregulated chemokine/CCL17 and macrophage-derived chemokine/CCL22 in epidermal keratinocytes and their roles in atopic dermatitis. Int. Immunol. 14, 767-773 (2002)

25. Ziegler, S. F. The role of thymic stromal lymphopoietin (TSLP) in allergic disorders. Curr. Opin. Immunol. 22, 795-799 (2010).

26. Kou, P. M. \& Babensee, J. E. Macrophage and dendritic cell phenotypic diversity in the context of biomaterials. J. Biomed. Mater. Res. A 96, 239-260 (2011).

27. Suarez, C. J., Parker, N. J. \& Finn, P. W. Innate immune mechanism in allergic asthma. Curr. Allergy Asthma Rep. 8, 451-459 (2008).

28. Yeh, K. W. et al. High cord blood CCL22/CXCL10 chemokine ratios precede allergic sensitization in early childhood. Oncotarget 8, 7384-7390 (2017).

29. Ait Yahia, S. et al. CCL17 production by dendritic cells is required for NOD1mediated exacerbation of allergic asthma. Am. J. Resp. Crit. Care 189, 899-908 (2014).

30. He, R. et al. TSLP acts on infiltrating effector $\mathrm{T}$ cells to drive allergic skin inflammation. Proc. Natl Acad. Sci. USA 105, 11875-11880 (2008).

31. Zhou, B. et al. Thymic stromal lymphopoietin as a key initiator of allergic airway inflammation in mice. Nat. Immunol. 6, 1047-1053 (2005).

32. Zheng, X. F. et al. Lipopolysaccharide-induced $M 2$ to $M 1$ macrophage transfor mation for IL-12p70 production is blocked by Candida albicans mediated upregulation of EBI3 expression. PLOS ONE 8, e63967 (2013).
33. Braga, T. T. et al. MyD88 signaling pathway is involved in renal fibrosis by favoring a TH2 immune response and activating alternative M2 macrophages. Mol. Med. 18, 1231-1239 (2012)

34. Stern, M. E. et al. Role of interferon-gamma in a mouse model of allergic conjunctivitis. Invest. Ophthalmol. Vis. Sci. 46, 3239-3246 (2005).

35. Magone, M. T., Chan, C. C., Rizzo, L. V., Kozhich, A. T. \& Whitcup, S. M. A novel murine model of allergic conjunctivitis. Clin. Immunol. Immunopathol. 87, 75-84 (1998).

36. Weischenfeldt, J. \& Porse, B. Bone Marrow-Derived Macrophages (BMM): Isolation and Applications. CSH Protoc. 2008, pdb.prot5080 (2008).

37. Marim, F. M., Silveira, T. N., Lima, D. S. Jr. \& Zamboni, D. S. A method for generation of bone marrow-derived macrophages from cryopreserved mouse bone marrow cells. PLOS ONE 5, e15263 (2010).

38. de Paiva, C. S. et al. IL-17 disrupts corneal barrier following desiccating stress. Mucosal Immunol. 2, 243-253 (2009).

39. Su, Z. et al. Potential autocrine regulation of interleukin-33/ST2 signaling of dendritic cells in allergic inflammation. Mucosal Immunol. 6, 921-930 (2013).

40. Bian, F. et al. Desiccating stress-induced MMP production and activity worsens wound healing in alkali-burned corneas. Invest. Ophthalmol. Vis. Sci. 56, 4908-4918 (2015).

41. Chi, W. et al. Mitochondrial DNA oxidation induces imbalanced activity of NLRP3/ NLRP6 inflammasomes by activation of caspase- 8 and BRCC36 in dry eye. J. Autoimmun. 80, 65-76 (2017)

42. Ma, P. et al. Human corneal epithelium-derived thymic stromal lymphopoietin links the innate and adaptive immune responses via TLRs and Th2 cytokines. Invest. Ophthalmol. Vis. Sci. 50, 2702-2709 (2009). 